Ann. Biol. anim. Bioch. Biophys., I075, 15 (I), г-8.

\title{
ÉTUDE COMPARÉE DU SÉRUM SANGUIN ET DU LIQUIDE FOLLICULAIRE PRÉOVULATOIRE CHEZ LA VACHE
}

\author{
Y. MENEZO et J. TESTART* \\ avec la collaboration technique de Chaqué Khatchadourian \\ Laboratoire de Biologie 406, I. N. R. A., \\ Institut national des Sciences appliquées, \\ 69621 Villeurbanne \\ * Station de Physiologie animale, \\ Centre national de Recherches zootechniques, I. N. R. A., \\ 78350 Jouy en Josas
}

\begin{abstract}
RÉSUMÉ
Le sérum et le liquide folliculaire préovulatoire de Vache ont été comparés selon quatre critères :

- les protéines étudiées par électrophorèse sur gel de polyacrylamide,

- les activités enzymatiques,

- la pression osmotique,

- les aminoacides libres.

Les diagrammes électrophorétiques folliculaires et sériques sont très voisins, l'albumine est le composé le plus important des deux liquides testés.

Les taux des différents amino acides sont très voisins dans les deux liquides; cependant la concentration totale est plus faible dans le liquide folliculaire.

En ce qui concerne les activités enzymatiques, la principale différence est liée à la présence dans le liquide folliculaire de $\mathrm{N}$-acétyl glucosaminidase et de $\beta$-glucuronidase, ces enzymes ne sont pas décelables dans le sérum.

La pression osmotique est légèrement plus faible dans le liquide folliculaire (288 milliosmoles) que dans le sérum (3ro milliosmoles).
\end{abstract}

\section{INTRODUCTION}

Le liquide folliculaire baigne l'ovocyte jusqu'au moment de l'ovulation et il est donc nécessaire d'en connaître la composition pour aborder in vitro l'étude de la maturation ovocytaire mais également de la fécondation dans la mesure où, chez les bovins, le volume de liquide émis par le follicule au moment de l'ovulation est très important. 
Les études chez la Vache (IUTWAK-MANN, I954; JENSFN et ZACHARIAE, I9.58; Desjardins et al., I 966), la Lapine (DAvid et al., I973), la 'Iruie (Shrvers et al., I964), la Femme (Shar,Gr et al., I973), ont montré que le sérum participe de façon très importante à l'élaboration du liquide folliculaire. Le rôle des protéines, des amino acides libres et de la pression osmotique (HAIDRI et Gwatkin, I973 ; Mryamoto et Chang, I973 $a, b, c)$ est primordial dans le processus de maturation ovocytaire et dans celui de la fécondation.

Ces trois paramètres et certaines activités enzymatiques ont été étudiés dans le sérum et le liquide folliculaire afin de mieux connaitre les relations existant au niveau de la paroi du follicule et également afin de déterminer si le sérum peut être employé pour l'élaboration des milieux de cultures de follicules et d'ovocytes.

\section{MATÉRIEL ET MÉTHODES}

\section{I. - Collecte des liquides}

Le liquide folliculaire est collecté sur cinq vaches, de race Française Frisonne Pie Noire, I 6 à 20 heures après le début des chaleurs, décelé par un taureau. A l'abattage, le liquide folliculaire du follicule le plus développé (environ $15 \mathrm{~mm}$ ) est prélevé directement à l'aide d'une seringue de $2,5 \mathrm{ml}$. Le sang des mêmes animaux est également collecté pour analyse du sérum.

\section{2. - Electrophorèses}

Les électrophorèses sont réalisées sur gel de polyacrylamide selon Davis (I964). Le sérum et le liquide folliculaire sont dilués au $\mathrm{I} / \mathrm{ro}$ avec du saccharose o,25 M avant dépôt. La coloration est réalisée à l'amido-Schwartz à I p. rooo dans l'acide acétique $7 \mathrm{p}$. Ioo. La décoloration est réalisée électrophorétiquement.

\section{3. - Zymogrammes}

Le dispositif employé ( ${ }^{1}$ ) est celui de Buissiz̀re et NARDon (I968). Il permet l'établissement des zymogrammes tissulaires et cellulaires et des liquides biologiques (PLANTEVin et Nardon, I972; FLEChON et MENEzo, 1973). Le sérum et le liquide folliculaire, placés en solutions tampons convenables sont incubés en présence d'un substrat chromogène sur un support de papier filtre placé dans un réservoir capillaire plastique. Après trois heures d'incubation à $37^{\circ} \mathrm{C}$, le résultat de la réaction enzymatique est estimé à l'œil nu par lecture de la coloration observée. La liste des I 8 enzymes testées et les substrats employés figurent dans le tableau I.

$$
\text { 4. - Pression osmotique }
$$

Les mesures sont faites directement à l'osmomètre Prolabo.

$$
\text { 5. - Acides aminés libres }
$$

Les analyses sont effectuées sur résine échangeuse d'ions (SPACKman et al., I958) sur aminolyzer Optica (Mondino, I968; Mondino et Bongiovanni, I972 ; Noe et al., I974; Menezo 1974). L'élution des amino acides neutres et acides est réalisée par des tampons au citrate de lithium de manière à obtenir une séparation quantitative de la glutamine et de l'asparagine. Les échantillons sont préparés de la façon suivante $: 0,5 \mathrm{ml}$ du liquide à analyser sont lyophilisés en présence de quantités connues de norleucine et d'acide $\alpha$-amino $\beta$-guanidino propionique utilisés comme standards internes. Le lyophilisat est repris à l'acide sulfosalycilique 3 p. Ioo selon Hamilton (I962), puis centrifugé. Le surnageant peut alors être utilisé directement pour analyse. L'étude de la reproductibilité indique que l'erreur n'est pas supérieure à 5 p. Ioo.

(1) API System, $3^{8-}$ La Balme les Grottes. 


\section{RÉSULTATS}

\section{I. - Électrophorèses (fig. I)}

Les diagrammes électrophorétiques ne font pas apparaître de différence entre les deux liquides testés où l'albumine et la transferrine sont les composés les plus importants.

Ces résultats sont en accord avec ceux de DESJARDins et al., (I966). Cependant, si les $\gamma$-globulines sont bien représentées dans le liquide folliculaire, la technique ne permet pas de déterminer si quelques composés sériques de hauts poids moléculaires en sont absents comme chez la Femme (Shalgi et al., I973).

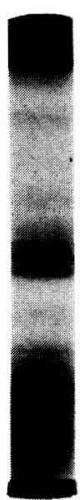

Sérum
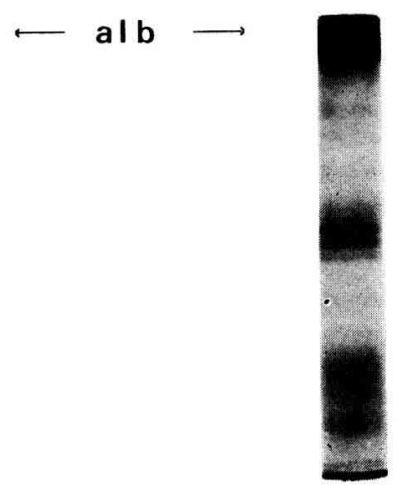

Liquide folliculaire

líg. I. - Electrophorèses sur gels de polyacrylamide

\section{2. - Zymogrammes (tabl. I)}

Les zymogrammes folliculaires et sériques sont assez voisins. Les activités phosphatasiques (déjà décrites dans le liquide folliculaire : KENNEY, I973), estérasiques et aminopeptidasiques, sont présentes dans les deux liquides. Une partie des enzymes repérées dans le follicule semble donc provenir du sérum, par filtration comme cela est observé pour les protéines non enzymatiques. Cependant, la différence la plus intéressante est observée pour les activités glucuronidasiques et $\mathrm{N}$-acétyl glucosaminidasiques, présentes dans le liquide folliculaire, mais non détectées dans le sérum. Il semble que ces deux activités soient équivalentes à la hyaluronidase responsable avec certaines enzymes protéolytiques de la rupture du follicule. Ces deux activités enzymatiques n'ont pas été détectées dans le liquide de follicules en voie d'atrésie $4^{0}$ à $5^{0}$ heures après le début des chaleurs. Sous réserve que cette activité hyaluronidasique n'apparaisse pas, ou soit inhibée, ou se trouve à un taux trop faible dans les follicules devant être frappés d'atrésie, la présence de cette enzyme pourrait être un critère de l'évolution normale du follicule, et par là même de l'ovocyte. La 
$\beta$-glucuronidase du liquide folliculaire pourrait intervenir dans le processus de capacitation des spermatozoïdes comme celà a été décrit chez le Hamster (Gwatkin et Hutchison, I97I).

TABLEAU I

Activités enzymatiques comparées du sérum et du liquide folliculaire

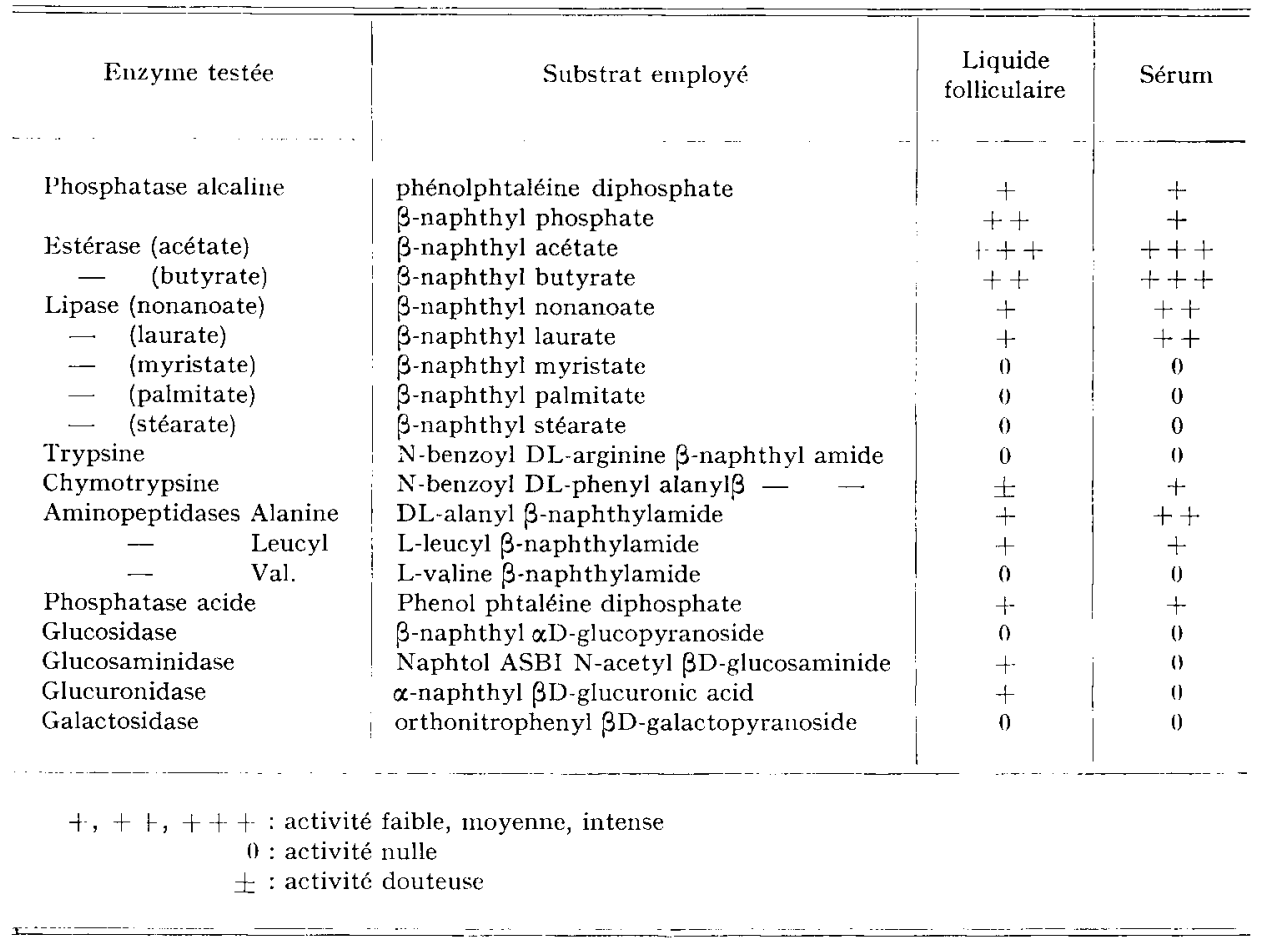

\section{3. - Pression osmotique}

La pression osmotique est, en accord avec Olds et VAN DEMaRK (I957), de 288 milliosmoles, dans le liquide folliculaire; elle est plus faible que celle du sérum : 3ro milliosmoles.

\section{4. - Amino acides libres (tabl. 2, fig. 2)}

Si la concentration totale en amino acides libres est légèrement plus faible dans le liquide folliculaire que dans le sérum, les taux relatifs des différents composés sont assez voisins dans les deux liquides. L'acide glutamique + glutamine, la glycine, l'alanine et la valine sont les composés les plus importants. Les quelques différences observées sont dues aux teneurs en taurine, glycine, ornithine et arginine, sensiblement plus faibles dans le liquide folliculaire que dans le sérum. I,es amino acides du cycle de l'urée semblent soit franchir difficilentent la paroi clu follicule, soit être métabolisés très rapidement à l'intérieur de celui-ci. 


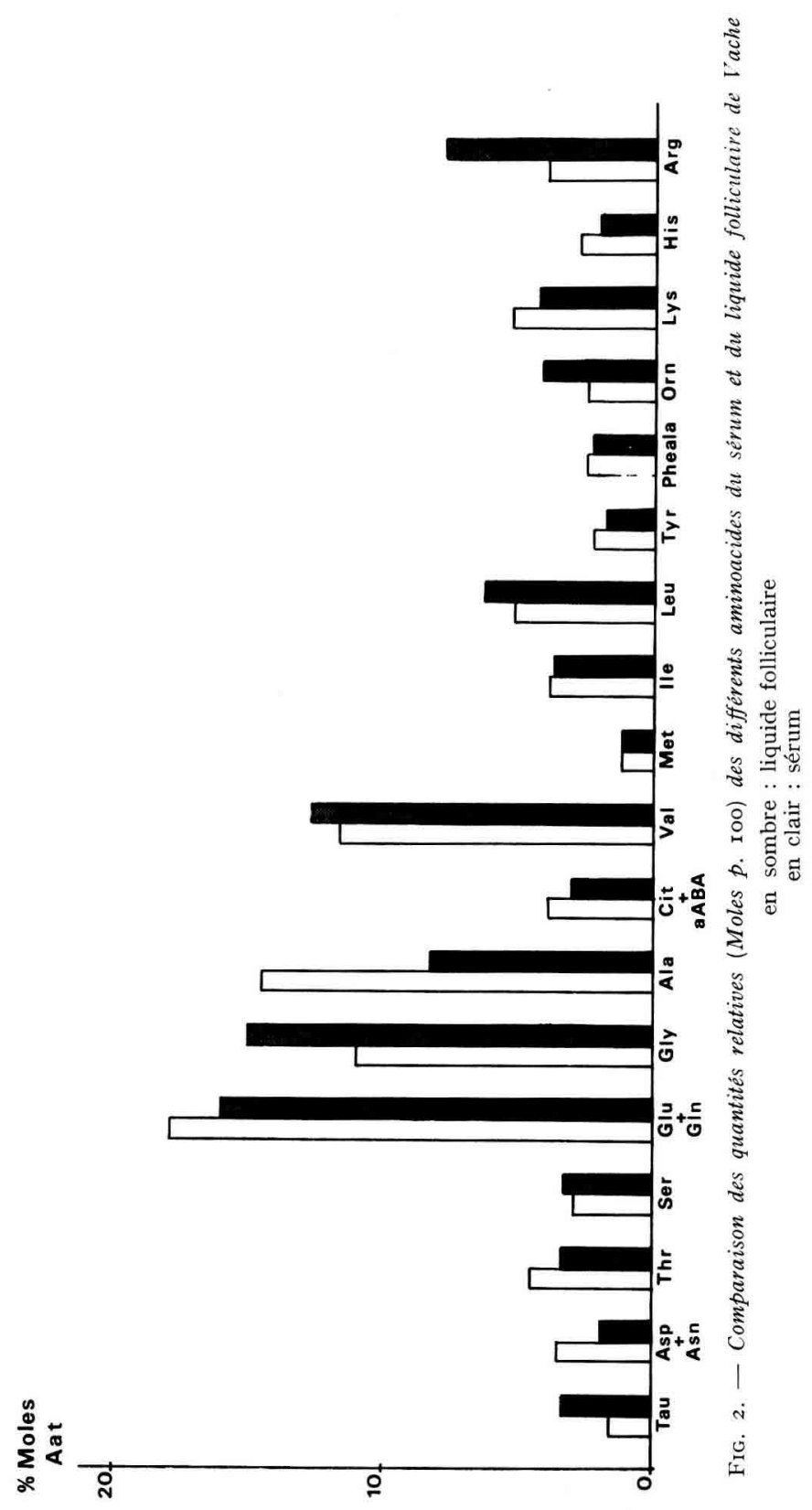




\section{TABLEAU 2}

Taux comparés des composés ninhidrine positifs du sérum sanguin et $d u$ liquide folliculaive de Vache ( $\mu \mathrm{M} / \mathrm{roo} \mathrm{ml})$

\begin{tabular}{|c|c|c|c|c|}
\hline \multirow{2}{*}{ Arides amines } & \multicolumn{2}{|c|}{ Liquide folliculaire } & \multicolumn{2}{|c|}{ Sérum } \\
\hline & $\mathrm{ml}$ & $\%$ & $\mathrm{~m}$ & $\%$ \\
\hline $\begin{array}{lll}-- & \cdot & \cdots\end{array}$ & $-\cdots---$ & $\cdots \cdots$ & - - & $\cdots-\cdots$ \\
\hline Taurine $\ldots \ldots \ldots \ldots$ & 3,1 & 1,5 & 9,8 & 3,9 \\
\hline Aspartique + Asp $\mathrm{NH}_{2} \ldots$ & 7,2 & 3,5 & 4,8 & 1,9 \\
\hline Thréonine $\ldots \ldots \ldots \ldots$ & 8,9 & 4,3 & 8,5 & 3,4 \\
\hline Sérine $\ldots \ldots \ldots \ldots \ldots$ & 6,1 & 2,9 & 8,2 & 3,2 \\
\hline Glutamique + Glu $\mathrm{NH}_{2}$. & 37,1 & 17,9 & 40,7 & 16,1 \\
\hline Glycocolle .......... & 22,7 & 11,0 & 37,7 & 14,9 \\
\hline Alanine...$\ldots \ldots \ldots$ & 29,9 & 14,5 & 20,9 & 8,3 \\
\hline $\begin{array}{l}\text { Citrulline }+ \text { acide } \alpha \text { ami- } \\
\text { nobutyrique } \ldots \ldots \ldots\end{array}$ & 8,1 & 3,9 & 7,5 & 3,0 \\
\hline Valine..$\ldots \ldots \ldots \ldots$ & 24,0 & 11,6 & 31,9 & 12,6 \\
\hline Méthionine . . . . . . . & 2,2 & 1,1 & 2,8 & 1,1 \\
\hline Isoleucine . . . . . . . . . . & 7,7 & 3,7 & 9,2 & 3,6 \\
\hline Leucine $\ldots \ldots \ldots \ldots \ldots$ & 10,6 & 5,1 & 15,4 & 6,1 \\
\hline Cystine . . . . . . . . & traces & & traces & \\
\hline Tyrosine $\ldots \ldots \ldots \ldots \ldots$ & 4,7 & 2,3 & 4,3 & 1,7 \\
\hline Phénylalanine $\ldots \ldots \ldots$ & 4,9 & 2,4 & 5,6 & 2,2 \\
\hline Ornithine $\ldots \ldots \ldots \ldots$ & 5,0 & 2,4 & 10,4 & 4,1 \\
\hline Lysine $\ldots \ldots \ldots \ldots \ldots$ & 11,0 & 5,3 & 10,9 & 4,3 \\
\hline Histidine $\ldots \ldots \ldots$ & 5,7 & 2,7 & 4,5 & 1,8 \\
\hline Arginine $\ldots . . .$. & 8,0 & 3,9 & 19,4 & 7,7 \\
\hline TOTAL & 206,9 & & 252,5 & \\
\hline
\end{tabular}

$\mathrm{m}:$ moyenne

$\%:$ pourcentage relatif $(\mu \mathrm{M} / 100)$

La concentration en alanine est par contre plus importante dans le liquide folliculaire que dans le sérum. HAIDRI et GWATKIN (I973) avaient observé que cet amino acide est indispensable à la maturation du follicule de Hamster in vitro.

Les résultats des analyses effectuées sur le sérum sont en accord avec ceux de FAhning et al., (I967) à ce stade du cycle sexuel. De même Desjardins et al., (I966) n'avaient pas observé de différences significatives entre le sérum et le liquide folliculaire pour l'azote aminé libre total.

\section{CONCLUSIONS}

Ces observations semblent favorables à l'utilisation du sérum dans les milieux de culture de follicules et éventuellement d'œufs fécondés. Cependant les différences observées au niveau de la pression osmotique et surtout la présence de la $\beta$-glucuronidase et la $\mathrm{N}$-acétyl glucosaminidase dans le liquide folliculaire indiquent claire- 
ment que celui-ci ne provient pas exclusivement d'une filtration de composés sériques. Il est en plus le résultat de l'activité hormonale et métabolique propre au follicule.

D'ailleurs Thibaurir (I966) avait obtenu des résultats nettement moins bons en cultivant l'œuf de Vache avec du sérum pur qu'avec du liquide folliculaire. Il semble donc que dans l'élaboration des milieux de culture on puisse utiliser pour partie du sérum, mais en tenant compte des faibles différences observées entre celui-ci et le liquide folliculaire et dont le rôle physiologique nous échappe pour l'instant.

\section{Reçu pour publication en janvier 1975.}

\section{SUMMARY}

\section{COMPARATIVE STUDY OF BLOOD SERUM AND PREOVULATORY FOLLICULAR FLUID OF THE COW}

Cow's blood serum and preovulatory follicular fluid were compared according to the following criteria :

- proteins studied by disc gel electrophoresis,

- enzymatic activities,

- osmolarity,

- free aminoacids.

- Disc gel electrophoresis do not show any difference between both fluids where albumin is the major component.

- The levels of the different aminoacids are not very different between the two fluids, however the total amount is slightly lower in follicular fluid.

- The most important difference in the enzymatic activities is due to the presence in folli cular fluid of $\mathrm{N}$-acetyl-glucosaminidase and glucuronidase, not detected in blood serum.

- Osmolarity is less important in follicular fluid ( 288 miliosmols) than in serum (3ro millios mols).

\section{RÉFÉRENCES BIBLIOGRAPHIQUES}

Buissière J., NARDoN P., I968. Microméthode d'identification des bactéries. I. Intérêt de la quantification des caractères biochimiques. Ann. Inst. Pasteur, 115, 218-23I.

David A., Frenkel G., Kraicer P. F., 1973. Chemical composition of rabbit follicular fluid. Fert. Steril., 24, 227-229.

Davis B. J., I964. Disc gel electrophoresis. IJ. Method and application to human serum proteins. AnnN. Y. Acad. Sci., 121, 404-427.

Desjardins C., Kirton K. T., Hafs H. D., I966. Some chemical, immunochemical and electrophoretic properties of bovine follicular fluid. J. Reprod. Fert., 11, 237-244.

Fahning M. L., Schultz R. H., Graham F. F., ig67. The free amino acid content of uterine fluids and blood serum in the cow. J. Reprod. Fert., 13, 229-236.

Gwatkin R. B. L., Hutchison C. F., i $97 x$. Hamster spermatozoa capacitated by glucuronidase. Nature, 187, $843-844$.

HAIDRI A. A., Gwatkin R. B. L., r973. Requirements for the maturation of Hamster oocytes from preovulatory follicles. J. Reprod. Fert., 3., I73-176.

Hamilton P. B., I962. Ion exchange chromatography of amino acids. Microdetermination of free amino acids in serum. Ann. N. Y. Acad. Sci., 102, 55-75.

Jensen C. E., Zachariae F., i 958. Studies on the mechanism of ovulation. Acta endocr., 29, 356-368.

KenNeY R. H., I973. Histoenzymologic evaluation of metabolic patterns in bovine follicular oocytes. An. J. Veter. Res., 34, 893-896.

Lutwak Mann C., I954. Note on the chemical composition of bovine follicular fluid. J. Agric. Sci., 44, $477-480$. 
MENEZO Y., Flechon J. E., r973. Utilisation d'une microtechnique pour la détermination des activités enzymatiques des spermatozoides et du plasma séminal chez le Lapin et le Taureau. C. R. Acad. Sci. Paris, sér. D, 277, 1037-1040.

Menezo Y., 1974. Les composés azotés des sécrétions tubaires et utérines chez la Brebis en oestrus. Comparaison avec le liquide séminal et le plasma sanguin. Coll. intern. Sperm transport and Fertilizing ability, I. N. S. E. R. M., 26, 389-406.

Miyamoto H., Chang M. C., I973a. Fertilization of rat eggs in vitro. Biol. Reprod., 9, 384-393.

Mryamoto $\mathrm{H}$., Chang M. C., I973 b. The importance of serum albumin and metabolic intermediates for capacitation of spermatozoa and fertilization of mouse eggs in vitro. J. Reprod. Fert., 32, I93-206.

Miyamoto H., Chang M. C., I973 c. Effect of osmolarity on fertilization of mouse and golden hamster eggs in vitro. J. Reprod. Fert., 33, 481-487.

Mondino A., I967. A new system for automatic aminoacid analysis. Part. I. J. Chromatog., 30, Ioo-II2.

Mondino A,, Bongiovani $G$., 1972. A rapid chromatographic determination of phenylalanine and tyrosine for phenyl ketonuria screening. J. chromatog., 69, 49-62.

Noe V., Menezo Y., Bonnot G., I974. Separazione di aminoacidi basici con tamponi al sodio su resina scambio ionica. Riv. Soc. Ital. Sc. Alimentazione, 6, I71-173.

Olds D., Van Demark N. L., 1957. Composition of luminal fluids in bovine female genitalia. Fert. Steril., 8, 345-35I.

Plantevin G., Nardon P., I972. Utilisation d'une microméthode de détection pour la recherche qualitative d'activités enzymatiques dans les tissus d'Insectes. Ann. Zool.-Écol. anim., 4, 229-248.

Shalgi R., Kraicer P. T., Rimon A., Pinto M., Soferman N., I973. Proteins of human follicular fluid : the blood follicle barrier. Fert. Stevil., 24, 429-434.

Shivers C. A., Metz C. B., Lutwak-ManN C., ig64. Some properties of pig follicular fluid. $J$. Reprod. Fert., 8, II5.

Spackman D. M., Stein W. H., Moore S., 1958. Automatic recording apparatus for use on the chromatography of amino acids. Anal. Chem., 32, I I go-I206.

Thibault C., I966. La culture in vitro de l'veuf de Vache. Ann. Biol. anim. Bioch. Biophys., 6, I59-I64. 